\title{
The effect of HF-rTMS over the left DLPFC on stress regulation as measured by Cortisol and Heart Rate Variability
}

\author{
Matias M. Pulopulos ${ }^{a}{ }^{*}$, Maximilian Schmausser ${ }^{\text {a }}$, Stefanie De Smet ${ }^{b c}$, \\ Marie-Anne Vanderhasselt ${ }^{a b c}$, Shishir Baliyan ${ }^{d}$, César Venero ${ }^{d}$, Chris Baeken ${ }^{b c e f}$, Rudi De Raedt ${ }^{a}$. \\ a Department of Experimental Clinical and Health Psychology, Ghent University, Belgium. \\ ${ }^{b}$ Department of Head and Skin, Ghent University, Belgium. \\ ${ }^{c}$ Ghent Experimental Psychiatry (GHEP) Lab, Belgium. \\ ${ }^{d}$ Department of Psychobiology, Universidad Nacional de Educación a Distancia (UNED), Spain. \\ e Department of Psychiatry, University Hospital Brussels (UZBrussel), Belgium. \\ ${ }^{f}$ Department of Electrical Engineering, Eindhoven University of Technology, The Netherlands.
}

Running title: HF-rTMS, left DLPFC and stress regulation.

* Corresponding author: Matias M. Pulopulos, Department of Experimental Clinical and Health Psychology, Ghent University, Henri Dunantlaan 2 - 9000 Ghent, Belgium. Tel: +32 (0)9 26464 72. Email: matias.pulopulos@ugent.be. 
Abstract

The prefrontal cortex, and especially the Dorsolateral Prefrontal Cortex (DLPFC), plays an inhibitory role in the regulation of the Hypothalamic-Pituitary-Adrenal (HPA) axis under stressful situations. Moreover, recent evidence suggests that a sustained DLPFC activation is associated with adaptive stress regulation in anticipation of a stressful event, leading to a reduced stress-induced amygdala response, and facilitating the confrontation with the stressor. However, studies using experimental manipulation of the activity of the DLPFC before a stressor are scarce, and more research is needed to understand the specific role of this brain area in the stress-induced physiological response. This preregistered study investigated the effect on stress regulation of a single excitatory high frequency (versus sham) repetitive transcranial magnetic stimulation (HF-rTMS) session over the left DLPFC applied before the Trier Social Stress Test in 75 healthy young women $(M=21.05, S D=2.60)$. Heart rate variability (HRV) and salivary cortisol were assessed throughout the experimental protocol. The active HF-rTMS and the sham group showed a similar cognitive appraisal of the stress task. No differences in HRV were observed during both the anticipation and the actual confrontation with the stress task and therefore, our results did not reflect DLPFC-related adaptive anticipatory adjustments. Importantly, participants in the active HF-rTMS group showed a lower cortisol response to the stress. The effect of left prefrontal HF-rTMS on the stress system provides further critical experimental evidence for the inhibitory role played by the DLPFC in the regulation of the HPA axis.

Keywords: HF-rTMS, HPA axis, Stress, Cortisol, HRV 


\section{Introduction}

Stressful events activate the hypothalamus-pituitary-adrenal (HPA) axis, resulting in an acute increase in glucocorticoids secretion (being cortisol the most important glucocorticoid in humans) (Dickerson and Kemeny, 2004). An exacerbated cortisol response to stress and the exposition to high levels of cortisol have been associated to the onset and maintenance of stress-related mental disorders such as anxiety, depression, and substance abuse (Faravelli et al., 2012; Herbert, 2013). Understanding the mechanisms involved in the regulation of the cortisol response to stress is crucial to comprehend, prevent and treat these stress-related health problems (McEwen, 2008).

The stress response is controlled by neurocircuitries connecting the prefrontal cortex and the amygdala (Ulrich-Lai and Herman, 2009). Particularly, it has been proposed that the dorsolateral prefrontal cortex (DLPFC) has indirect inhibitory projections to the amygdala (e.g., Drevets, 1999; Chen et al., 2018; Siegle et al., 2007), a subcortical region that has excitatory connections with the HPA axis through projections to the hypothalamus (Herman et al., 2005). Along this line, previous research observed that manipulating the activity of the DLPFC using repetitive transcranial magnetic stimulation (rTMS) does not modulate the emotional stress reactivity (Remue et al., 2016a; Smits et al., In press), but it improves the regulation of the physiological response to psychosocial stressors (Baeken et al., 2014; Remue et al., 2016b; although no effects on a pharmacological stressor have been observed Zwanzger et al., 2007). Baeken et al. (2014) showed that increasing the activity of the left DLPFC using high frequency repetitive transcranial magnetic stimulation (HF-rTMS) attenuates the HPA response to a paradigm designed to induce a moderated level of anxiety and stress using bogus negative feedback on the participants' performance on a mental counting task. Using the same task, Remue et al. (2016b) observed that after receiving HF-rTMS over the left DLPFC participants did not show a stressinduced decrease in Heart Rate Variability (HRV), a physiological marker that reflects the inhibitory control of the prefrontal cortex over the activity of the heart via the vagus nerve, the main component of the parasympathetic nervous system (see Fagundes et al., 2011; Thayer et al., 2012 for a detailed 
description). These results suggest that the left DLPFC plays an important role in the regulation of the physiological response to stress. However, it is important to note that the task used in Baeken et al. (2014) and Remue et al. (2016b) did not provoke changes in mood, indicating that the task may not have been perceived as stressful by the participants. In this regard, Antal et al. (2014) investigated the effect on the cortisol response of transcranial direct current stimulation (tDCS) over the right medial prefrontal cortex before the Trier Social Stress Task (TSST; Kirschbaum et al., 1993), a stress task that provokes a robust physiological and psychological stress response in humans (Allen et al., 2017). They showed that anodal tDCS (to increase cortical excitability) and cathodal tDCS (to reduce cortical excitability) reduced and increased cortisol levels after the TSST, respectively. Although these results support the idea that the prefrontal cortex participates in the regulation of the HPA response to a psychosocial stressor, this study did not manipulate DLPFC activity specifically. Moreover, in contrast to rTMS, tDCS stimulates broad cortical regions with lower spatial precision, and it manipulates the membrane potential of neurons, but it does not directly activate the neurons (Paulus, 2011; Thielscher et al., 2015). Therefore, an important gap in the literature is whether rTMS over the DLPFC before stress could also affect the regulation of the HPA axis when individuals are confronted with a significant psychological stressful situation. Additionally, more research is needed to understand the specific role of this brain area in the stress-induced cortisol response.

In the Neurocognitive Framework for Regulation Expectation, De Raedt and Hooley, (2016) proposed that the influence of the DLPFC on the stress response starts even before the stressful event occurs, i.e. during the anticipation of the stressful situation. It is important to note that stress-related physiological changes do not only occur during the exposure to an acute stressor but also as a result of stress anticipation (Engert et al., 2013; Pulopulos et al., 2020, 2018b). To this regard, it has been proposed that during stress anticipation individuals make behavioral, cognitive, and physiological adjustments (i.e., anticipatory stress regulation) that facilitate the confrontation with the upcoming actual stressor (Nasso et al., 2019; Pulopulos et al., 2020, 2018b; Schulkin, 2011; Turan, 2015; Turan et 
al., 2015). Following the Neurocognitive Framework for Regulation Expectation (De Raedt and Hooley, 2016), these anticipatory stress regulation processes depend on the maintenance of goal-relevant information associated with a sustained activation of the DLPFC. Importantly, via the cortical-subcortical pathway, the sustained activation of the DLPFC would reduce the activation of the amygdala and will facilitate the process of stress regulation before and during the confrontation with the stressor. In line with this idea, in two studies assessing anticipatory stress regulation via changes in the vagally-mediated $\mathrm{HRV}$, we have recently observed that better anticipatory stress regulation is associated with an attenuated cortisol response to stress (Pulopulos et al., 2020, 2018b). Thus, we could expect that increased DLPFC activity during stress anticipation would be associated with enhanced anticipatory stress regulation (i.e. reflected in lower decreases in HRV) and an attenuated HPA axis response to stress (i.e. reflected in lower cortisol increase).

To examine this important question, this study aims to investigate for the first time whether HF-rTMS over the left DLPFC affects the cortisol and HRV response when it is applied immediately before the Trier Social Stress Test (TSST; Kirschbaum et al., 1993), a stress task that provokes a robust physiological and psychological stress response in humans (Allen et al., 2017), and that allows for the investigation of anticipatory stress regulation. We hypothesized that participants receiving active HFrTMS will show an attenuated cortisol response to the TSST compared to the participants in the sham group. Moreover, we expect that the active HF-rTMS group will show a lower decrease in HRV during anticipation (reflecting better anticipatory stress regulation), and during the stress task (reflecting better reactive stress regulation).

The hypotheses of this study, together with a detailed description of the sampling criteria, methodology, and analysis pipeline, were pre-registered on the Open Science Framework (see https://osf.io/tv47g).

\section{Methods}

\subsection{Participants}


In a between-subject design, 80 healthy right-handed females between 18 and 35 years old were recruited for this study. After the exclusion of some participants, the final sample included in the analyses were 75 participants (see Data management and statistical analyses section for a detailed description of the exclusion of participants). In order to control for the effects of sex and menstrual cycle on the cortisol response to stress (Pulopulos et al., 2018a), only women were included in this study, and the number of participants taking hormonal contraceptives and in the luteal and follicular phase of their menstrual cycle were counterbalanced between groups. Most participants were taking hormonal contraceptives (Hormonal contraceptives $=62$, Luteal $=7$, Follicular $=5)^{1}$, and there were no differences between groups in the number of participants taking hormonal contraceptives and in the luteal or follicular phase of the menstrual cycle $\left(X^{2}=1.50, p=0.475\right)$. The exclusion criteria were: smoking more than 10 cigarettes per day, presence of psychiatric disorders, cardiovascular disorders, use of medication that may affect cortisol levels, cognitive and cardiovascular activity (e.g., glucocorticoids, psychotropic substances, beta-blockers, sleep medications, etc), inner ear prosthesis, recent neurosurgical procedures, pacemaker or other electronic implants, metal objects or magnetic objects in the brain or around the head (only removable earrings and piercings were allowed), pregnancy, skin disorders at the level of the head.

\section{Baseline questionnaires}

The participants were asked to complete a series of questionnaires assessing depression symptoms (Beck Depression Inventory-II; Beck et al. 1996; Dutch version: Van der Does, 2002), selfesteem (Rosenberg Self-Esteem Scale; Rosenberg, 1965; Dutch version: Franck et al., 2008), trait and state anxiety (State-Trait Anxiety Inventory; Spielberger et al., 1970; Dutch version: Van der Ploeg, 1980), rumination (Rumination Response Scale; Nolen-Hoeksema and Morrow, 1991); Dutch version:

\footnotetext{
${ }^{1}$ The statistical conclusions remain the same if only participants taking hormonal contraceptives are included in the analyses.
} 
Raes et al., 2003), and perceived stress (Perceived Stress Scale; Cohen et al., 1983) to explore differences between groups since these variables may affect the cortisol response to stress and the effects of rTMS on cortisol secretion (e.g., Baeken et al., 2011; Chopra et al., 2009; De Witte et al., 2020; Pruessner et al., 2005; Puig-Perez et al., 2016; Salvador and Costa, 2009; Shull et al., 2016; VrshekSchallhorn et al., 2018; Zoccola and Dickerson, 2012). The data for baseline questionnaires could not be recorded for one participant.

\subsection{Anticipatory cognitive appraisal}

Stress appraisal was measured at the beginning of the anticipation period to the TSST using a Dutch version of the Primary Appraisal Secondary Appraisal scale (PASA; Gaab et al., 2005), a 16 items questionnaire, rated on a 6-point Likert scale (ranging from 1=Totally disagree, to 6=Totally agree). The PASA allows for the calculation of three subscales assessing "Primary appraisal” (i.e., how threatful and challenging the situation is perceived), "Secondary appraisal" (i.e., Self-concept of own competence and control expectancy), and a global index of "Anticipatory stress appraisal" (calculated as Primary appraisal minus Secondary appraisal) which sets the two appraisals in relation to each other. See Gaab et al. (2005) for a detailed description of the questionnaire.

\subsection{Physiological response to stress}

Heart Rate Variability

Heart rate data was continuously recorded throughout the session using a telemetric heart rate monitor (Polar v800) with a Polar H10 heart rate sensor and a pro chest strap placed just below the participant's chest muscles. The resulting beat-to-beat intervals were imported to Kubios 3.0.2 (Tarvainen et al., 2014, 2002) for artefacts correction and R-to-R intervals interpolation. The HRV values from two participants of each group could not be obtained due to problems with the heart rate monitor. As an index of vagally-mediated HRV, we used the root mean square successive difference (RMSSD), considered an index of stress regulation (Fagundes et al., 2011; Thayer et al., 2012) that is relatively free of respiratory influences (Laborde et al., 2017). 
Cortisol

Salivary samples were collected using Salivettes (Sarstedt, Nümbrecht, Germany) to assess acute changes in cortisol levels. Participants were instructed to keep the cotton swab in their mouths for $2 \mathrm{~min}$ and move the swab around in a circular pattern to collect saliva from all salivary glands. The

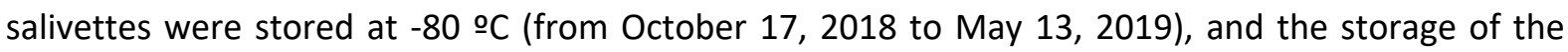
samples was operationally managed by the Bioresource Center Ghent (Ghent, Belgium, ID: BE 71067049; see T'Joen et al., 2018). The samples were analyzed in duplicate at the laboratory of Professor Venero at the Universidad Nacional de Educación a Distancia (Spain) using a commercially available enzyme-linked immunosorbent assay (Salimetrics ${ }^{\circledR}$ ) with an expected lower detection limit of $0.007 \mu \mathrm{g} / \mathrm{dL}$ and intra- and inter-assay precision of 2.5 and $4.7 \%$.

\subsection{Stress task}

The TSST (Kirschbaum et al., 1993) was used to induce stress. The TSST comprises of three consecutive parts. The first part is an introduction to the task, in which the participants are informed about the task that they have to perform. They are informed that they have to give a 5-min speech explaining why they believe they are the best candidate for a job position of their choice, and that after the speech they will perform a difficult arithmetic task. The second part is the anticipatory phase, in which participants have time to prepare their speech. Finally, the last part is the 5-min speech and a 5-min arithmetic task (counting backwards from 2083 to zero in 13-step sequences). A modified version of the TSST was used for this study. First, given that we are interested in regulatory mechanisms that occur during stress anticipation, the anticipatory phase lasted 15 minutes. Moreover, we used a one-member committee. Previous studies have shown a significant physiological and psychological response to the TSST using a 15 min anticipation and using only one-member committee (e.g., Engert et al., 2013; Pulopulos et al., 2020; Zandara et al., 2016).

\subsection{Neurostimulation}


The stimulation was performed over left DLPFC using a Magstim Rapid² Plus1 stimulator (Magstim Company Limited, Minneapolis, USA) with an eight-shaped coil, and using a stimulation intensity of $110 \%$ of the subject's resting motor threshold. The resting motor threshold was calculated immediately before the stimulation, and it was operationalized as the minimum TMS intensity necessary to yield a motor response in the right abductor pollicis brevis muscle in 5 out of 10 successive attempts. The high-frequency $(20 \mathrm{~Hz})$ stimulation sessions consisted of 40 trains of $2 \mathrm{~s}$ duration, separated by an intertrain interval of $12 \mathrm{~s}$ (1600 pulses per session). Participants in the sham group were exposed to a sham stimulation using the Magstim $70 \mathrm{~mm}$ Double Air Film sham coil, a coil that mimics the physical and auditory sensation of a real stimulation, and that looks exactly like the active coil. The active and sham coils were positioned over the left DLPFC using the adjusted BeamF3 algorithm (Mir-Moghtadaei et al., 2015). All participants were blindfolded and had ear protection during the stimulation.

\subsection{Procedure}

The participants provided written informed consent at the beginning of the experiment. The study has been approved by the Ethical Committee of the University Hospital of Ghent University (UZ Gent; Belgian registration number: B670201837272). This study was carried out in compliance with the declaration of Helsinki.

The participants were recruited to participate in an experiment investigating the role of the DLPFC in social interactions. The recruitment of participants was performed via SONA (Experiment Management System) and internet posts. They attended an individual session for the induction of stress response. They were asked to sleep as long as usual and to abstain from alcohol and heavy physical exercise for 24 hours before the session, and to drink only water, not eat, smoke, brush their teeth or take any stimulants (such as coffee, cola, caffeine, tea or chocolate) from two hours before the session. At the beginning of the session, the participants were asked to stay quiet in the room for $25 \mathrm{~min}$ to habituate to the laboratory. During the last $10 \mathrm{~min}$ of habituation, participants completed some of the baseline questionnaires (i.e., State version of the State-Trait Anxiety Inventory, Perceived 
Stress Scale, and Beck Depression Inventory II). After the habituation period, participants received the active HF-rTMS or sham stimulation. Five min after the stimulation, they were introduced to the TSST task, and they were asked to prepare the task for $15 \mathrm{~min}$ (i.e., anticipatory phase). At the beginning of the anticipation phase, they completed the PASA to assess cognitive stress appraisal. After 15 min of anticipation, they performed the speech $(5 \mathrm{~min}$ ) and the arithmetic task ( $5 \mathrm{~min}$ ) of the TSST. After the stress task, the participants were asked to stay quiet for $30 \mathrm{~min}$. During the last $5 \mathrm{~min}$ of the recovery phase, they were asked to complete the Rosenberg Self-Esteem Scale and the Rumination Response Scale. At the end of the session, the participants' height and weight was measured to compute the body mass index. On a different day, they completed the Temperament and Character Inventory (results not reported here) and the trait version of the State-Trait Anxiety Inventory. Finally, the participants were debriefed about the purpose of the study and received 20 euros for their participation. Although HF-rTMS was applied before the TSST, previous research has shown that the effect may last for up to 60 min (Hoogendam et al., 2010; Thut and Pascual-Leone 2008), and therefore, the stimulation is expected to affect how the participants anticipate and perform the TSST.

The participant provided five saliva samples to measure cortisol levels: (i) at the end of the habituation period, (ii) after the sham or active HF-rTMS session, and then (iii) $20 \mathrm{~min}$, (iv) $30 \mathrm{~min}$, and (v) 40 min after the onset of the speech task. The participants were asked to fill in four 10-centimetres Visual Analogue Scales (VAS; McCormack et al., 1988) to assess stress perception, tiredness, happiness, and tension. The VASs were completed: (i) at the end of the habituation phase, (ii) after the sham or active HF-rTMS session, (iii) at the end of the anticipatory phase, (iv) after the speech task, (v) immediately after the arithmetic task, and (vi) 40min after the onset of the stress task. See Figure 1 for an overview of these measures during the protocol.

To control for the circadian rhythm of cortisol secretion, the experimental sessions started at $14.00 \mathrm{~h}$ or at $16.00 \mathrm{~h}$. The starting time was counterbalanced between groups, and there were no significant differences between groups in the time when they started the session $\left(X^{2}=6.51, p=0.420\right)$. 


\subsection{Data management and statistical analyses}

For HRV, the last $10 \mathrm{~min}$ of the habituation and the last $10 \mathrm{~min}$ of the anticipation were separated into 5 min epochs and averaged to compute the HRV levels at baseline and during anticipation, respectively. HRV levels during the speech $(5 \mathrm{~min}$ ) and the arithmetic $(5 \mathrm{~min}$ ) tasks were averaged to compute the HRV during the stress task. For cortisol levels, two indexes were computed and used as dependent variables for the analyses: (i) The area under the curve with respect to the increase (AUCi) was computed using the formula proposed by Pruessner et al. (2003) as a measure of dynamic of the cortisol change from baseline (using the samples collected immediately before the TSST, and every 10 min after the TSST) to after the TSST. (ii) The cortisol reactivity was calculated as the change in cortisol from baseline (immediately before the TSST) to the maximum cortisol levels after the stress task, a measure of the magnitude of the cortisol response provoked by the stress task.

$T$-tests were used to investigate differences between groups (sham vs. active HF-rTMS) in demographics, baseline questionnaires, cognitive stress appraisal (i.e., Primary Appraisal, Secondary Appraisal, and Anticipatory stress appraisal), and cortisol indexes (i.e., cortisol AUCi and cortisol reactivity). Mixed MANOVAs were used to investigate the psychological response to stress using Group (sham vs. active HF-rTMS) as a between-subject factor and Time (Before rTMS, Before TSST, End of anticipation, After speech, After math, and $\mathbf{3 0}$ min after the TSST) as a within-subject factor. The four VAS mood scales were the multiple dependent variables. Finally, mixed ANOVA was used to investigate changes in HRV using Group (sham vs. active HF-rTMS) as a between-subject factor and Time (Habituation, Anticipation, and Stress) $)^{2}$ as a within-subject factor. Where necessary, we applied the Greenhouse-Geisser correction to ensure the assumption of sphericity. Cortisol, HRV and VAS data, and the distribution of the residuals of the ANOVAs did not follow a normal distribution. Therefore, cortisol,

\footnotetext{
${ }^{2}$ Given our research question, we did not include HRV levels during the recovery period in the analyses. However, the statistical conclusions are the same if HRV during the recovery period is included in the mixed ANOVA investigating changes in HVR levels.
} 
HRV and VAS data were log transformed. The log transformation of these variables resulted in a normalization of the distribution of the residuals for cortisol and HRV, but not for VAS data.

Eighty women were recruited for this study. However, one participant ate $30 \mathrm{~min}$ before the session, one participant was sick, and one participant had several job interviews during the days previous to the experiment. These participants were excluded from the study. Moreover, two participants were excluded from the analyses because they were outliers (3SD) for cortisol (one participant in the active HF-rTMS group) and HRV (one participant in the active HF-rTMS group) data. Therefore, the final sample was composed of 37 participants in the active HF-rTMS group, and 38 participants in the sham group.

Analyses were performed using SPSS 24.0 (IBM SPSS Statistics 24.0), and the significance level was set at $p=0.05$, two-tailed, for all analyses.

\section{Results}

\subsection{Demographics and baseline questionnaires}

Table 1 shows the characteristics of the study sample. No significant differences between groups (sham vs active HF-rTMS) were observed in age, body mass index, subjective socioeconomic status (assessed using the subjective socioeconomic status scale: Adler et al., 2000), and their scores in depression symptoms, rumination, perceived stress in the past month, state and trait anxiety, and self-esteem (all $p \mathrm{~s}>0.131)$.

\subsection{Anticipatory cognitive appraisal}

The results of the $t$-tests showed no differences between groups in primary $(t(72)=0.65$, $p=0.517, d=0.17)$, secondary $(t(72)=0.30, p=0.762, d=0.08)$, and anticipatory stress appraisal $(t(63.45)=0.60, p=0.550, d=0.14)$ (See Table 1$).$

\subsection{Psychological response to stress}


The repeated measures MANOVA revealed a significant multivariate effect of Time (Pillai's Trace $\left.=0.77, F(20,53)=8.99, p<0.001, \eta p^{2}=0.05\right)$. The factor Group and the interaction between Group and Time were not statistically significant (Group: Pillai's Trace $=0.05, F(4,69)=0.92, p=0.459, \eta p^{2}=0.77$; Group*Time: Pillai's Trace $\left.=0.28, F(20,53)=1.00, p=0.475, n p^{2}=0.28\right)$. Follow-up ANOVAs showed that the factor Time was statistically significant for the four subscales 'stress' $(F(2.69,193.91)=42.90$, $\left.p<0.001, \quad \eta p^{2}=0.37\right), \quad$ tension' $\quad\left(F(3.21,230.74)=36.34, \quad p<0.001, \quad \eta p^{2}=0.34\right), \quad$ tiredness' $\left(F(3.89,280.33)=3.03, p=0.019, \eta p^{2}=0.04\right)$, and 'happiness' $\left(F(4.03,289.78)=31.83, p<0.001, \eta p^{2}=0.31\right)$. Overall, participants reported being significantly less stressed $(p=0.024)$, and slightly less tense $(p=0.059)$ after the active or sham stimulation than before the stimulation. The participants also reported being more stressed, tense and less happy during stress anticipation, after the speech task, and after the arithmetic task than immediately after the active or sham stimulation (all $p s<0.003$ ). Moreover, they reported being less happy at the end of the recovery phase than immediately before the stress task (i.e., after the active or sham stimulation) $(p=0.008)$. No significant changes in 'tiredness' were observed (all ps>0.082). See Table 2 for the VAS scores.

\subsection{HRV response}

Figure 2 shows HRV levels during habituation, anticipation, and the stress task for the sham and active HF-rTMS groups. The ANOVA for repeated measured showed a significant effect of Time $\left(F(1.52,104.54)=69.17, p<0.001, n p^{2}=0.50\right) . H R V$ showed a significant decrease from the habituation phase to the anticipation of the stress task ( $\left.p=0.007, d_{a v}=0.25\right)$, and from the anticipation phase to the stress task $\left(p=0.001, d_{a v}=0.82\right)$. The factor Group (sham vs active HF-rTMS) $(F(1,69)=0.11, p=0.747$, $\left.\eta p^{2}<0.01\right)$, and the interaction between Time and Group were not statistically significant $\left(F(1.52,104.54)=0.12, p=0.831, n p^{2}<0.01\right)$.

\subsection{Cortisol response to stress}


The $t$-test showed higher $A U C i$ in the sham than in the active HF-rTMS group $(t(73)=2.08$, $p=0.041, d=0.48$; Figure $3 \mathrm{~A})$. Regarding cortisol reactivity, the sham group also showed higher values than the active HF-rTMS, however, the difference was not statistically significant $(t(73)=1.47, p=0.146$, $d=0.33$; Figure 3B). See Table 2 for the cortisol levels at each time point during the session for the sham and active HF-rTMS groups.

\section{Discussion}

This study investigated for the first time the effects of a single excitatory rTMS session applied to the left DLPFC before the TSST on the HRV and cortisol response to stress. The active HF-rTMS and the sham group showed a similar anticipatory cognitive appraisal of the stress task, and no differences in stress-induced changes in HRV and mood were observed. Importantly, participants in the active HFrTMS group showed a lower cortisol response to stress than participants in the sham group.

This study provides experimental evidence for the role of the left DLPFC in the neurobiological modulation of the HPA axis. In accordance with our hypothesis, we observed that a single HF-rTMS session over the left DLPFC attenuates the cortisol response to stress, an effect reflected in AUCi values. For cortisol reactivity, the sham group also showed higher values than the active HF-rTMS group, but the difference was not statistically significant. These results suggest that HF-rTMS over the left DLPFC would especially affect the sensitivity of the HPA axis to a challenge, reflected in AUCi values (Fekedulegn et al., 2007) but not the magnitude of the cortisol increase, reflected in the cortisol reactivity index (Lopez-Duran et al., 2014). Moreover, given that the AUCi is a measure of the pattern of cortisol changes over time, it also reflects the duration of the HPA axis response to stress (i.e., longer HPA axis response would be reflected in higher AUCi values). Thus, our results are also in line with Ulrich-Lai and Herman (2009), who proposed that the prefrontal cortex regulates the duration but not the peak levels of the cortisol secretion. However, given that cortisol levels peak between 20 and 40 min after the stressor onset, the smaller differences in cortisol reactivity may also be due to the fact 
that, for some participants, we did not capture the cortisol peak because it occurred between the three samples collected after the TSST (Dickerson and Kemeny, 2004; Lopez-Duran et al., 2014).

Our results are in line with a previous study showing a reduced cortisol response (reflected in lower AUCi values) in healthy females receiving DLPFC HF-rTMS before being exposed to a critical feedback paradigm (Baeken et al., 2014). Yet, unlike Baeken et al. (2014), the stress paradigm used in the current study provoked a robust psychological response. Together, these results indicate that the effect of HF-rTMS over the left DLPFC on HPA axis activity can be generalized to stressors of different magnitude characterized by social-evaluative threat and uncontrollability. Importantly, in line with a recent review and a meta-analysis (Remue et al., 2016a; Smits et al., In press), the stimulation did not affect the psychological response to stress, and therefore our results cannot be attributed to an influence of HF-rTMS in mood. Morevover, given that both sham and active stimulation groups showed similar anticipatory cognitive appraisal, this study further demonstrates that an increased DLPFC activity affects the HPA axis response to stress independently of the appraisal of the stressful situation and of the ability to deal with it. It is well known that under stressful situations the amygdala is activated and initiates the response of the HPA axis (Herman et al., 2005), and studies in animals have demonstrated that the prefrontal cortex has an inhibitory role in the regulation of the HPA axis (Gilabert-Juan et al., 2013; Herman et al., 2003). Moreover, several studies suggest that the DLPFC participates in emotion regulation via indirect inhibitory connections to the amygdala (e.g., Drevets, 1999; Chen et al., 2018; Siegle et al., 2007). Together, the current evidence supports the idea that the DLPFC plays a central role in the regulatory processes of the HPA axis when individuals are confronted with psychosocial stressors.

In line with neuroimaging data showing prefrontal abnormalities (predominantly on the left) in patients with unipolar depression (e.g., Drevets et al., 2008), HF-rTMS over the left DLPFC has been used as an effective treatment for major depression (see De Raedt et al., 2015; Baeken and De Raedt, 2011). However, the underlying working mechanisms are still not fully understood. Within this 
context, our results provide more evidence to understand the processes underlying the therapeutic effect of rTMS. Exposure to high levels of cortisol may damage the prefrontal cortex (Lupien et al., 2007), and is associated with decreased DLPFC activity and sustained activation of the amygdala, a dysregulation that may lead to an exacerbated HPA axis response to daily stressors, and a subsequent reduced ability to regulate emotions (De Raedt and Koster, 2010). It has been proposed that the therapeutic effects of multiple rTMS sessions could be related to a restoration of the regulation of the HPA system (Baeken and De Raedt, 2011; Keck, 2003; Schutter and van Honk, 2010). Besides reducing the stress-induced HPA axis response, as observed in the current study and in Baeken et al. (2014), excitatory rTMS over the left DLPFC may also reduce the overall cortisol secretion when applied in individuals after being stressed (Pulopulos et al., 2019). Additionally, a single session of active HF-rTMS over the left DLPFC and not sham reduces cortisol secretion in depressed patients (Baeken et al., 2009). Importantly, Drysdale et al. (2017) recently showed that the functional connectivity between the left DLPFC and several brain regions including the left amygdala predicts responsiveness to rTMS therapy in depressed patients. Together, these observations suggest that, by increasing left prefrontal cortex activity, excitatory rTMS would improve the ability of the system to regulate the HPA axis when confronted with psychological stressors. In the long-term, this would reduce brain exposure to the pernicious effects of high cortisol levels due to stress, an effect that would prevent prefrontal cortex damage and contribute to a restoration of the inhibitory control of the prefrontal cortex activity over the amygdala.

We hypothesized that the active HF-rTMS group would show a lower decrease in the vagallymediated HRV during anticipation, and during the speech and math tasks, reflecting better anticipatory and reactive stress regulation, respectively. In contrast to our hypothesis, no differences between groups were observed. Our results suggest that HF-rTMS did not improve DLPFC-related adaptive anticipatory adjustments in our participants. One possible explanation is that an increase of DLPFC activity would improve anticipatory stress regulation only if the individuals engage in these behavioral 
and cognitive adjustments (using an adaptive emotion regulation strategy) during stress anticipation. Unfortunately, we did not measure the use of stress regulation strategies during the anticipation phase. Further studies are needed to investigate whether HF-rTMS over the DLPFC, together with anticipatory stress regulation strategies affects HRV levels and have a more substantial effect on the cortisol response to stress. Importantly, our results are also in contrast to previous studies showing an effect of HF-rTMS (Remue et al., 2016b) and anodal transcranial direct current stimulation (Carnevali et al., 2020) over the left DLPFC on the HRV levels during stress. It is noteworthy that, in these studies, the active stimulation condition showed an increase (Carnevali et al., 2020; Remue et al., 2016b) or a small decrease (Carnevali et al., 2020) in HRV levels during the task. In the current study, a significant decrease in HRV was observed in both groups (which is the regular HRV response to a stressor), pointing to inconsistent results. A plausible explanation for the different results could also be related to the characteristics of the stress task. In contrast to Remue et al. (2016b) and Carnevali et al. (2019), the task used in the current study involved physical activity (participants had to walk from one room to another to receive the instructions and to perform the stress task), variation in body posture (participants were standing when performing the stress task), and more mental workload (participants had to give a 5-min structured speech), three factors associated with changes in the activity of the sympathetic and parasympathetic nervous system (Hjortskov et al., 2004; Houtveen et al., 2005; Pulopulos et al., 2013; Wiemers et al., 2013). Thus, it is possible that, although HF-rTMS activated DLPFC-related regulatory process, some components of the task might have masked the subtle differences in HRV associated with differences in stress regulation processes in the sham versus the real stimulation condition.

Despite the methodological strengths of this preregistered study, it should be noted that although the stress task provoked a robust psychological and cardiovascular response to stress, it only provoked a low cortisol increase in the sham group. This result can be explained by the fact that most participants were taking hormonal contraceptives and that a relatively small increase in cortisol levels 
is commonly observed in this population (for a review see Pulopulos et al., 2018a). This result can be explained by the fact that the levels of bioavailable free cortisol in women taking hormonal contraceptives, as can be measured in salivary samples, are lower than the total cortisol secretion (see (Kudielka et al., 2009). More studies are needed to investigate whether similar effects are observed in men and in women in the luteal and follicular phase of the menstrual cycle. Finally, we observed small to medium effect sizes for the effect of HF-rTMS on the cortisol response (i.e., AUCi: $d=0.48$, Cortisol reactivity: $d=0.33$ ). These results might be explained by the relatively high interindividual variability of the modulatory effects of rTMS on cortical excitability (Maeda et al., 2000; Nettekoven et al., 2015). Future research should consider larger sample sizes when investigating the effect of rTMS on stress regulation.

In conclusion, the results of this preregistered study provide the first evidence for the effect of a left DLPFC HF-rTMS session before the TSST on stress regulation. Although HF-rTMS did not affect HRV, our observations indicate that by increasing the activity of the left DLPFC, we can enhance the regulation of the stress-induced changes in HPA axis activity. The effect on the HPA axis may have important implications for the treatment of affective disorders.

\section{Acknowledgment}

The authors would like to thank Daphne Van der Meulen and Jozefien Tilleman for their help during data collection. This research was supported by Grant BOF16/GOA/017 for a Concerted Research Action of Ghent University (awarded to RDR and CB), by Grant RTI2018-094627-B-I00 from the Spanish Ministry of Economy and Competitivity (awarded to CV), by Grant BOFSTA2017002501 for research at Ghent University (awarded to MAV) and by the bilateral research Grant GOF4619N from the Research Foundation Flanders (awarded to MAV). MMP is a postdoctoral research fellow, supported by the Research Foundation Flanders (FWO18/PDO/174).

\section{References}

Adler, N.E., Epel, E.S., Castellazzo, G., Ickovics, J.R., 2000. Relationship of subjective and objective social status with psychological and physiological functioning: Preliminary data in healthy, White women. Health Psychology 19, 586-592. https://doi.org/10.1037/0278-6133.19.6.586 
Allen, A.P., Kennedy, P.J., Dockray, S., Cryan, J.F., Dinan, T.G., Clarke, G., 2017. The Trier Social Stress Test: Principles and practice. Neurobiology of Stress 6, 113-126. https://doi.org/10.1016/j.ynstr.2016.11.001

Baeken, C., De Raedt, R. 2011. Neurobiological mechanisms of repetitive transcranial magnetic stimulation on the underlying neuro circuitry in unipolar depression. Dialogues in clinical neuroscience 13(1), 139.

Baeken, C., De Raedt, R., Leyman, L., Schiettecatte, J., Kaufman, L., Poppe, K., Vanderhasselt, M.A., Anckaert, E., Bossuyt, A., 2009. The impact of one HF-rTMS session on mood and salivary cortisol in treatment resistant unipolar melancholic depressed patients. Journal of Affective Disorders 113(1-2), 100-108. https://doi.org/10.1016/j.jad.2008.05.008

Baeken, C., Vanderhasselt, M.A., De Raedt, R., 2011. Baseline 'state anxiety' influences HPA-axis sensitivity to one sham-controlled HF-rTMS session applied to the right dorsolateral prefrontal cortex. Psychoneuroendocrinology 36, 60-67. https://doi.org/10.1016/j.psyneuen.2010.06.006

Baeken, C., Vanderhasselt, M.A., Remue, J., Rossi, V., Schiettecatte, J., Anckaert, E., De Raedt, R., 2014. One left dorsolateral prefrontal cortical HF-rTMS session attenuates HPA-system sensitivity to critical feedback in healthy females. Neuropsychologia 57, 112-121. https://doi.org/10.1016/j.neuropsychologia.2014.02.019

Beck, A.T., Steer, R.A., Brown, G. 1996. Beck Depression Inventory-II. San Antonio.

Carnevali, L., Pattini, E., Sgoifo, A., Ottaviani, C., 2020. Effects of prefrontal transcranial direct current stimulation on autonomic and neuroendocrine responses to psychosocial stress in healthy humans. Stress 23, 26-36. https://doi.org/10.1080/10253890.2019.1625884

Chen, F., Ke, J., Qi, R., Xu, Q., Zhong, Y., Liu, T., Li, J., Zhang, L., Lu, G. 2018. Increased inhibition of the amygdala by the mPFC may reflect a resilience factor in post-traumatic stress disorder: A 
resting-state fMRI Granger causality analysis. Frontiers in psychiatry, 9, 516.

https://doi.org/10.3389/fpsyt.2018.00516

Chopra, K.K., Ravindran, A., Kennedy, S.H., Mackenzie, B., Matthews, S., Anisman, H., Bagby, R.M., Farvolden, P., Levitan, R.D., 2009. Sex differences in hormonal responses to a social stressor in chronic major depression. Psychoneuroendocrinology 34, 12351241.https://doi.org/10.1016/j.psyneuen.2009.03.014

Cohen, S., Kamarck, T., Mermelstein, R., 1983. A global measure of perceived stress. J. Health Soc. Behav. 24, 385-396. https://doi.org/10.2307/2136404.

De Raedt, R., Hooley, J.M., 2016. The role of expectancy and proactive control in stress regulation: A neurocognitive framework for regulation expectation. Clinical Psychology Review 45, 45-55. https://doi.org/10.1016/j.cpr.2016.03.005

De Raedt, R., Vanderhasselt, M. A., \& Baeken, C. (2015). Neurostimulation as an intervention for treatment resistant depression: From research on mechanisms towards targeted neurocognitive strategies. Clinical Psychology Review, 41, 61-69.

https://doi.org/10.1016/j.cpr.2014.10.006

De Raedt, R., Koster, E.H.W., 2010. Understanding vulnerability for depression from a cognitive neuroscience perspective: A reappraisal of attentional factors and a new conceptual framework. Cognitive, Affective, \& Behavioral Neuroscience 10, 50-70.

https://doi.org/10.3758/CABN.10.1.50

De Witte, S., Baeken, C., Pulopulos, M.M., Josephy, H., Schiettecatte, J., Anckaert, E., De Raedt, R., Vanderhasselt, M.-A., 2020. The effect of neurostimulation applied to the left dorsolateral prefrontal cortex on post-stress adaptation as a function of depressive brooding. Progress in Neuro-Psychopharmacology and Biological Psychiatry 96, 109687. https://doi.org/10.1016/j.pnpbp.2019.109687 
Drevets, W.C. 1999. Prefrontal cortical-amygdalar metabolism in major depression. Ann N Y Acad Sci. 877, 614-637. https://doi.org/10.1111/j.1749-6632.1999.tb09292.x

Drevets, W.C., Price, J.L., Furey, M.L. 2008. Brain structural and functional abnormalities in mood disorders: implications for neurocircuitry models of depression. Brain structure and function, 213(1-2), 93-118. https://doi.org/10.1007/s00429-008-0189-x

Dickerson, S.S., Kemeny, M.E., 2004. Acute Stressors and Cortisol Responses: A Theoretical Integration and Synthesis of Laboratory Research. Psychological Bulletin 130, 355-391. https://doi.org/10.1037/0033-2909.130.3.355

Engert, V., Efanov, S.I., Duchesne, A., Vogel, S., Corbo, V., Pruessner, J.C., 2013. Differentiating anticipatory from reactive cortisol responses to psychosocial stress. Psychoneuroendocrinology 38, 1328-1337. https://doi.org/10.1016/j.psyneuen.2012.11.018

Fagundes, C.P., Murray, D.M., Hwang, B.S., Gouin, J.-P., Thayer, J.F., Sollers, J.J., Shapiro, C.L., Malarkey, W.B., Kiecolt-Glaser, J.K., 2011. Sympathetic and parasympathetic activity in cancer-related fatigue: More evidence for a physiological substrate in cancer survivors. Psychoneuroendocrinology 36, 1137-1147. https://doi.org/10.1016/j.psyneuen.2011.02.005

Faravelli, C., Lo Sauro, C., Lelli, L., Pietrini, F., Lazzeretti, L., Godini, L., Benni, L., Fioravanti, G., Alina Talamba, G., Castellini, G., Ricca, V., 2012. The Role of Life Events and HPA Axis in Anxiety Disorders: A Review. CPD 18, 5663-5674. https://doi.org/10.2174/138161212803530907

Franck, E., De Raedt, R., Barbez, C., Rosseel, Y., 2008. Psychometric Properties of the Dutch Rosenberg Self-Esteem Scale. PSYCHOL BELG 48, 25. https://doi.org/10.5334/pb-48-1-25

Gaab, J., Rohleder, N., Nater, U.M., Ehlert, U., 2005. Psychological determinants of the cortisol stress response: the role of anticipatory cognitive appraisal. Psychoneuroendocrinology 30, 599-610. https://doi.org/10.1016/j.psyneuen.2005.02.001 
Gilabert-Juan, J., Castillo-Gomez, E., Guirado, R., Moltó, M.D., Nacher, J., 2013. Chronic stress alters inhibitory networks in the medial prefrontal cortex of adult mice. Brain Struct Funct 218, 1591-1605. https://doi.org/10.1007/s00429-012-0479-1

Herbert, J., 2013. Cortisol and depression: three questions for psychiatry. Psychol. Med. 43, 449469. https://doi.org/10.1017/S0033291712000955

Herman, J.P., Figueiredo, H., Mueller, N.K., Ulrich-Lai, Y., Ostrander, M.M., Choi, D.C., Cullinan, W.E., 2003. Central mechanisms of stress integration: hierarchical circuitry controlling hypothalamo-pituitary-adrenocortical responsiveness. Frontiers in Neuroendocrinology 24, 151-180. https://doi.org/10.1016/j.yfrne.2003.07.001

Herman, J.P., Ostrander, M.M., Mueller, N.K., Figueiredo, H., 2005. Limbic system mechanisms of stress regulation: Hypothalamo-pituitary-adrenocortical axis. Progress in Neuro-Psychopharmacology and Biological Psychiatry 29, 1201-1213. https://doi.org/10.1016/j.pnpbp.2005.08.006

Hjortskov, N., Riss n, D., Blangsted, A.K., Fallentin, N., Lundberg, U., S gaard, K., 2004. The effect of mental stress on heart rate variability and blood pressure during computer work. European Journal of Applied Physiology 92, 84-89. https://doi.org/10.1007/s00421-004-1055-z

Hoogendam, J.M., Ramakers, G. M., Di Lazzaro, V., 2010. Physiology of repetitive transcranial magnetic stimulation of the human brain. Brain stimulation 3(2), 95-118.

https://doi.org/10.1016/j.brs.2009.10.005

Houtveen, J.H., Groot, P.F.C., Geus, E.J.C., 2005. Effects of variation in posture and respiration on RSA and pre-ejection period. Psychophysiology 42, 713-719. https://doi.org/10.1111/j.14698986.2005.00363.x

Keck, M.E., 2003. rTMS as treatment strategy in psychiatric disorders-neurobiological concepts, in: Supplements to Clinical Neurophysiology. Elsevier, pp. 100-116. 
Kirschbaum, C., Pirke, K.-M., Hellhammer, D.H., 1993. The 'Trier Social Stress Test' - A Tool for Investigating Psychobiological Stress Responses in a Laboratory Setting. Neuropsychobiology 28, 76-81. https://doi.org/10.1159/000119004

Kudielka, B.M., Hellhammer, D.H., Wüst, S., 2009. Why do we respond so differently? Reviewing determinants of human salivary cortisol responses to challenge. Psychoneuroendocrinology 34 , 2-18. https://doi.org/10.1016/j.psyneuen.2008.10.004

Laborde, S., Mosley, E., Thayer, J.F., 2017. Heart Rate Variability and Cardiac Vagal Tone in Psychophysiological Research - Recommendations for Experiment Planning, Data Analysis, and Data Reporting. Front. Psychol. 08. https://doi.org/10.3389/fpsyg.2017.00213

Lopez-Duran, N.L., Mayer, S.E., Abelson, J.L., 2014. Modeling neuroendocrine stress reactivity in salivary cortisol: adjusting for peak latency variability. Stress 17(4), 285-295. https://doi.org/10.3109/10253890.2014.915517

Lupien, S.J., Maheu, F., Tu, M., Fiocco, A., Schramek, T.E., 2007. The effects of stress and stress hormones on human cognition: Implications for the field of brain and cognition. Brain and Cognition 65, 209-237. https://doi.org/10.1016/j.bandc.2007.02.007

Maeda, F., Keenan, J.P., Tormos, J.M., Topka, H., Pascual-Leone, A., 2000. Interindividual variability of the modulatory effects of repetitive transcranial magnetic stimulation on cortical excitability. Experimental Brain Research 133(4), 425-430. https://doi.org/10.1007/s002210000432

McCormack, H.M., de L. Horne, D.J., Sheather, S., 1988. Clinical applications of visual analogue scales: a critical review. Psychol. Med. 18, 1007-1019.

https://doi.org/10.1017/\$0033291700009934

McEwen, B.S., 2008. Central effects of stress hormones in health and disease: Understanding the protective and damaging effects of stress and stress mediators. European Journal of Pharmacology 583, 174-185. https://doi.org/10.1016/j.ejphar.2007.11.071 
Mir-Moghtadaei, A., Caballero, R., Fried, P., Fox, M.D., Lee, K., Giacobbe, P., Daskalakis, Z.J., Blumberger, D.M., Downar, J., 2015. Concordance Between BeamF3 and MRI-neuronavigated Target Sites for Repetitive Transcranial Magnetic Stimulation of the Left Dorsolateral Prefrontal Cortex. Brain Stimulation 8, 965-973. https://doi.org/10.1016/j.brs.2015.05.008

Nasso, S., Vanderhasselt, M.-A., Demeyer, I., De Raedt, R., 2019. Autonomic regulation in response to stress: The influence of anticipatory emotion regulation strategies and trait rumination. Emotion 19, 443-454. https://doi.org/10.1037/emo0000448

Nolen-Hoeksema, S., Morrow, J., 1991. A prospective study of depression and posttraumatic stress symptoms after a natural disaster: The 1989 Loma Prieta earthquake. Journal of Personality and Social Psychology 61, 115-121. https://doi.org/10.1037/0022-3514.61.1.115

Nettekoven, C., Volz, L.J., Leimbach, M., Pool, E.M., Rehme, A.K., Eickhoff, S.B., Fink, G.R., Grefkes, C., 2015. Inter-individual variability in cortical excitability and motor network connectivity following multiple blocks of rTMS. Neuroimage 118, 209-218 https://doi.org/10.1016/j.neuroimage.2015.06.004

Pruessner, J.C., Baldwin, M.W., Dedovic, K., Renwick, R., Mahani, N.K., Lord, C., Meaney, M., Lupien, S., 2005. Self-esteem, locus of control, hippocampal volume, and cortisol regulation in young and old adulthood. Neurolmage $28,815-826$. https://doi.org/10.1016/j.neuroimage.2005.06.014

Pruessner, J.C., Kirschbaum, C., Meinlschmid, G., Hellhammer, D.H., 2003. Two formulas for computation of the area under the curve represent measures of total hormone concentration versus time-dependent change. Psychoneuroendocrinology 28, 916-931. https://doi.org/10.1016/S0306-4530(02)00108-7

Puig-Perez, S., Villada, C., Pulopulos, M.M., Hidalgo, V., Salvador, A., 2016. How are neuroticism and depression related to the psychophysiological stress response to acute stress in healthy older people? Physiology \& Behavior 156, 128-136. https://doi.org/10.1016/j.physbeh.2016.01.015 
Pulopulos, M.M., Almela, M., Hidalgo, V., Villada, C., Puig-Perez, S., Salvador, A., 2013. Acute stress does not impair long-term memory retrieval in older people. Neurobiology of Learning and Memory 104, 16-24. https://doi.org/10.1016/j.nIm.2013.04.010

Pulopulos, M.M., Baeken, C., De Raedt, R., 2020. Cortisol response to stress: The role of expectancy and anticipatory stress regulation. Hormones and Behavior 117, 104587. https://doi.org/10.1016/j.yhbeh.2019.104587

Pulopulos, M.M., De Witte, S., Vanderhasselt, M.-A., De Raedt, R., Schiettecatte, J., Anckaert, E., Salvador, A., Baeken, C., 2019. The influence of personality on the effect of iTBS after being stressed on cortisol secretion. PLoS ONE 14, e0223927. https://doi.org/10.1371/journal.pone.0223927

Pulopulos, M.M., Hidalgo, V., Puig-Pérez, S., 2018a. Psychophysiological response to social stressors: Relevance of sex and age. Psicothema 171-176. https://doi.org/10.7334/psicothema2017.200 Pulopulos, M.M., Vanderhasselt, M.-A., De Raedt, R., 2018b. Association between changes in heart rate variability during the anticipation of a stressful situation and the stress-induced cortisol response. Psychoneuroendocrinology 94, 63-71. https://doi.org/10.1016/j.psyneuen.2018.05.004

Raes, F., Hermans, D., Eelen, P. 2003. De Nederlandstalige versie van de Ruminative Response Scale en de Rumination on Sadness Scale (The Dutch version of the Rumination Response Scale and the Rumination on Sadness Scale). Gedragstherapie, 36, 97-104.

Remue, J., Baeken, C., De Raedt, R., 2016a. Does a single neurostimulation session really affect mood in healthy individuals? A systematic review. Neuropsychologia 85, 184-98. https://doi.org/10.1016/j.neuropsychologia.2016.03.012.

Remue, J., Vanderhasselt, M.-A., Baeken, C., Rossi, V., Tullo, J., De Raedt, R., 2016b. The effect of a single HF-rTMS session over the left DLPFC on the physiological stress response as measured by heart rate variability. Neuropsychology 30, 756-766. https://doi.org/10.1037/neu0000255 
Rosenberg, M. 1965. Society and the Adolescent Self-Image. Princeton, NJ: Princeton University Press.

Salvador, A., Costa, R., 2009. Coping with competition: Neuroendocrine responses and cognitive variables. Neuroscience \& Biobehavioral Reviews 33, 160-170. https://doi.org/10.1016/j.neubiorev.2008.09.005

Schulkin, J., 2011. Social Allostasis: Anticipatory Regulation of the Internal Milieu. Front. Evol. Neurosci 2. https://doi.org/10.3389/fnevo.2010.00111

Schutter, D.J.L.G., van Honk, J., 2010. An endocrine perspective on the role of steroid hormones in the antidepressant treatment efficacy of transcranial magnetic stimulation. Psychoneuroendocrinology 35, 171-178. https://doi.org/10.1016/j.psyneuen.2009.04.009

Shull, A., Mayer, S.E., McGinnis, E., Geiss, E., Vargas, I., Lopez-Duran, N.L., 2016. Trait and state rumination interact to prolong cortisol activation to psychosocial stress in females. Psychoneuroendocrinology 74, 324-332. https://doi.org/10.1016/j.psyneuen.2016.09.004

Siegle, G.J., Thompson, W., Carter, C.S., Steinhauer, S.R., Thase, M.E. 2007. Increased amygdala and decreased dorsolateral prefrontal BOLD responses in unipolar depression: related and independent features. Biological Psychiatry 61(2), 198-209. https://doi.org/10.1016/j.biopsych.2006.05.048

Spielberger, C.D., Gorsuch, R.L., Lushene, R.E. 1970. Manual for the State-Trait Anxiety Inventory. Consulting Psychologist Press, Palo Alto.

Smits, F.M., Schutter, D.J.L.G, van Honk, J., Geuze, E., Does non-invasive brain stimulation modulate emotional stress reactivity?, Social Cognitive and Affective Neuroscience In press https://doi.org/10.1093/scan/nsaa011

Tarvainen, M.P., Niskanen, J.-P., Lipponen, J.A., Ranta-aho, P.O., Karjalainen, P.A., 2014. Kubios HRV - Heart rate variability analysis software. Computer Methods and Programs in Biomedicine 113, 210-220. https://doi.org/10.1016/j.cmpb.2013.07.024 
Tarvainen, M.P., Ranta-aho, P.O., Karjalainen, P.A., 2002. An advanced detrending method with application to HRV analysis. IEEE Trans. Biomed. Eng. 49, 172-175.

https://doi.org/10.1109/10.979357

Thayer, J.F., Åhs, F., Fredrikson, M., Sollers, J.J., Wager, T.D., 2012. A meta-analysis of heart rate variability and neuroimaging studies: Implications for heart rate variability as a marker of stress and health. Neuroscience \& Biobehavioral Reviews 36, 747-756.

https://doi.org/10.1016/j.neubiorev.2011.11.009

Thielscher, A., Antunes, A., Saturnino, G.B., 2015. Field modeling for transcranial magnetic stimulation: a useful tool to understand the physiological effects of TMS?, In: 2015 37th Annual International Conference of the IEEE Engineering in Medicine and Biology Society (EMBC). IEEE, 222-5. https://doi.org/10.1109/EMBC.2015.7318340.

Thut, G., Pascual-Leone, A., 2010. A review of combined TMS-EEG studies to characterize lasting effects of repetitive TMS and assess their usefulness in cognitive and clinical neuroscience. Brain topography 22(4), 219. https://doi.org/10.1007/s10548-009-0115-4

T’Joen, V., Phlypo, S., Bekaert, S., 2018. Bimetra Biobank: A High Quality Biobank Facility to Stimulate Translational Biomedical Research. Open Journal of Bioresources 5, 7. https://doi.org/10.5334/ojb.37

Turan, B., 2015. Predictors of anticipatory cortisol reactivity to subsequent stressors. Physiology \& Behavior 149, 239-246. https://doi.org/10.1016/j.physbeh.2015.06.011

Turan, B., Foltz, C., Cavanagh, J.F., Alan Wallace, B., Cullen, M., Rosenberg, E.L., Jennings, P.A., Ekman, P., Kemeny, M.E., 2015. Anticipatory sensitization to repeated stressors: The role of initial cortisol reactivity and meditation/emotion skills training. Psychoneuroendocrinology 52, 229-238. https://doi.org/10.1016/j.psyneuen.2014.11.014

Ulrich-lai, Y., \& Herman, J.P. 2009. Neural regulation of endocrine and autonomic stress responses. Nature Reviews Neuroscience 10(6), 397-409. http://dx.doi.org/10.1038/nrn2647 
Van der Does, A.J.W. 2002. Manual of the Dutch version of the BDI-II. San Antonio, TX/Lisse, Ed.

Van der Ploeg, H.M. 1980. Validity of the Zelf-Beoordelings-Vragenlijst (A Dutch version of the Spielberger State-Trait Anxiety Inventory). Nederlands Tijdschrift voor de Psychologie en haar Grensgebieden.

Vrshek-Schallhorn, S., Avery, B.M., Ditcheva, M., Sapuram, V.R., 2018. The cortisol reactivity threshold model: Direction of trait rumination and cortisol reactivity association varies with stressor severity. Psychoneuroendocrinology 92, 113-122.

https://doi.org/10.1016/j.psyneuen.2017.11.002

Wiemers, U.S., Schoofs, D., Wolf, O.T., 2013. A friendly version of the Trier Social Stress Test does not activate the HPA axis in healthy men and women. Stress 16, 254-260.

https://doi.org/10.3109/10253890.2012.714427

Zandara, M., Garcia-Lluch, M., Pulopulos, M.M., Hidalgo, V., Villada, C., Salvador, A., 2016. Acute stress and working memory: The role of sex and cognitive stress appraisal. Physiology \& Behavior 164, 336-344. https://doi.org/10.1016/j.physbeh.2016.06.022

Zoccola, P.M., Dickerson, S.S., 2012. Assessing the relationship between rumination and cortisol: A review. Journal of Psychosomatic Research 73, 1-9. https://doi.org/10.1016/j.jpsychores.2012.03.007 


\section{Legends}

Figure 1. Overview of the psychological and physiological measures of the stress response during the session. See Procedure for a detailed description of the timing of these measures. TSST $=$ Trier Social Stress Test; HF-rTMS = High Frequency repetitive Transcranial Magnetic Stimulation; PASA = Primary and Secondary Appraisal Scale; VAS = Visual Analogue Scale; HRV = Heart Rate variability.

Figure 2. Heart rate variability (RMSSD) during the habituation, stress anticipation, and the stress task for the Active HF-rTMS and sham groups. Means and standard errors. ${ }^{*} p<0.05$.

Figure 3. (Fig. A) AUCi for the active and sham HF-rTMS groups. (Fig. B) Cortisol reactivity for the active and sham HF-rTMS groups. ${ }^{*} p<0.05$. 
Table 1. Characteristics of the study sample and scores in anticipatory cognitive appraisal.

\begin{tabular}{lrrrc}
\hline & $\begin{array}{c}\text { Active HF-rTMS } \\
\text { Mean (SD) }\end{array}$ & \multicolumn{1}{c}{ Sham } & & \\
& \multicolumn{1}{c}{ Mean (SD) } & \multicolumn{1}{c}{$t$} & \multicolumn{1}{c}{$p$} \\
\cline { 2 - 5 } Age & $20.86(2.91)$ & $21.24(2.30)$ & 0.62 & 0.540 \\
Body mass index $\left(\mathrm{kg} / \mathrm{cm}^{2}\right)$ & $22.70(4.31)$ & $23.00(3.66)$ & 0.33 & 0.744 \\
Subjective socioeconomic status & $5.81(1.00)$ & $6.16(0.97)$ & 1.53 & 0.131 \\
Perceived Stress Scale & $21.64(7.45)$ & $21.74(6.80)$ & 0.06 & 0.953 \\
Rosenberg Self-Esteem Questionnaire & $9.72(2.89)$ & $10.26(2.59)$ & 0.85 & 0.399 \\
Rumination Response Scale & $19.83(12.40)$ & $23.13(10.35)$ & 1.25 & 0.217 \\
Beck Depression Inventory II & $7.47(6.11)$ & $5.87(5.15)$ & 1.22 & 0.225 \\
STAI State & $46.33(3.44)$ & $47.47(3.78)$ & 1.35 & 0.180 \\
STAI Trait & $46.49(4.04)$ & $47.34(3.74)$ & 0.95 & 0.344 \\
Primary appraisal & $4.25(0.86)$ & $4.13(0.70)$ & 0.65 & 0.517 \\
Secondary appraisal & $3.68(0.74)$ & $3.73(0.56)$ & 0.30 & 0.762 \\
Anticipatory stress appraisal & $0.56(1.34)$ & $0.40(0.97)$ & 0.60 & 0.550 \\
\hline
\end{tabular}

Note: HF-rTMS=High Frequency repetitive Transcranial Magnetic Stimulation 
Table 2. Mean (SD) for VAS and cortisol levels during the session.

\begin{tabular}{|c|c|c|c|c|c|c|c|c|c|}
\hline \multirow{2}{*}{$\begin{array}{l}\text { VAS } \\
\text { Stress }\end{array}$} & Group & Before rTMS & Before TSST & End of & \multicolumn{2}{|c|}{ After Speech } & \multicolumn{2}{|c|}{ After Math } & $\begin{array}{c}30 \text { min after } \\
\text { TSST } \\
\end{array}$ \\
\hline & Active & $21.69(18.17)$ & $15.51(15.41)$ & 63.09 (19.81) & \multicolumn{2}{|c|}{73.10 (23.19) } & \multicolumn{2}{|c|}{$43.36(29.96)$} & $19.28(23.25)$ \\
\hline & Sham & $21.72(22.34)$ & $14.75(19.26)$ & $56.34(22.63)$ & \multicolumn{2}{|c|}{$62.27(24.70)$} & \multicolumn{2}{|c|}{37.45 (24.19) } & 13.81 (16.69) \\
\hline \multirow[t]{2}{*}{ Tension } & Active & $19.01(18.16)$ & $19.55(20.40)$ & $58.32(25.39)$ & \multicolumn{2}{|c|}{$67.51(24.48)$} & \multicolumn{2}{|c|}{$42.58(30.20)$} & $18.44(21.45)$ \\
\hline & Sham & $23.06(24.80)$ & $16.40(21.12)$ & $55.40(22.71)$ & \multicolumn{2}{|c|}{$61.78(24.96)$} & \multicolumn{2}{|c|}{$41.55(26.17)$} & $18.47(21.10)$ \\
\hline \multirow[t]{2}{*}{ Tiredness } & Active & $35.93(21.72)$ & $36.68(24.70)$ & $32.68(27.22)$ & \multicolumn{2}{|c|}{$29.09(26.72)$} & \multicolumn{2}{|c|}{$29.24(25.52)$} & $39.46(25.17)$ \\
\hline & Sham & $29.87(22.43)$ & 35.26 (22.99) & $32.18(23.47)$ & \multicolumn{2}{|c|}{$27.64(21.23)$} & \multicolumn{2}{|c|}{$28.18(20.13)$} & $38.96(25.68)$ \\
\hline \multirow[t]{2}{*}{ Happiness } & Active & $67.96(14.25)$ & $64.22(20.23)$ & $47.29(22.20)$ & \multicolumn{2}{|c|}{38.13 (24.89) } & \multicolumn{2}{|c|}{$51.69(27.70)$} & $59.65(21.38)$ \\
\hline & Sham & $64.59(12.90)$ & 60.57 (17.64) & $45.65(20.64)$ & \multicolumn{2}{|c|}{39.67 (23.64) } & \multicolumn{2}{|c|}{$45.05(24.80)$} & 52.35 (18.49) \\
\hline \multirow{3}{*}{$\begin{array}{l}\text { Cortisol } \\
\text { (ug/L) }\end{array}$} & & Before rTMS & Before TSST & \multicolumn{2}{|c|}{10 min after TSST } & \multicolumn{2}{|c|}{20 min after TSST } & \multicolumn{2}{|c|}{30 min after TSST } \\
\hline & Active & $0.181(0.073)$ & $0.179(0.081)$ & \multicolumn{2}{|c|}{$0.162(0.083)$} & \multicolumn{2}{|c|}{$0.163(0.105)$} & \multicolumn{2}{|c|}{$0.147(0.078)$} \\
\hline & Sham & $0.170(0.102)$ & $0.153(0.078)$ & $0.163(c$ & 113) & 0.17 & (0.123) & & $148(0.106)$ \\
\hline
\end{tabular}

Note: VAS = the Visual Analogue Scale; TSST = Trier Social Stress Test. 
Figure 1

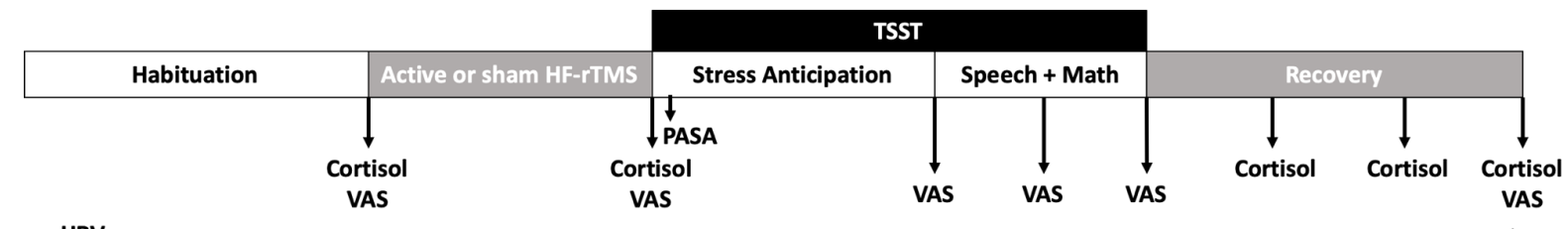

Figure 2

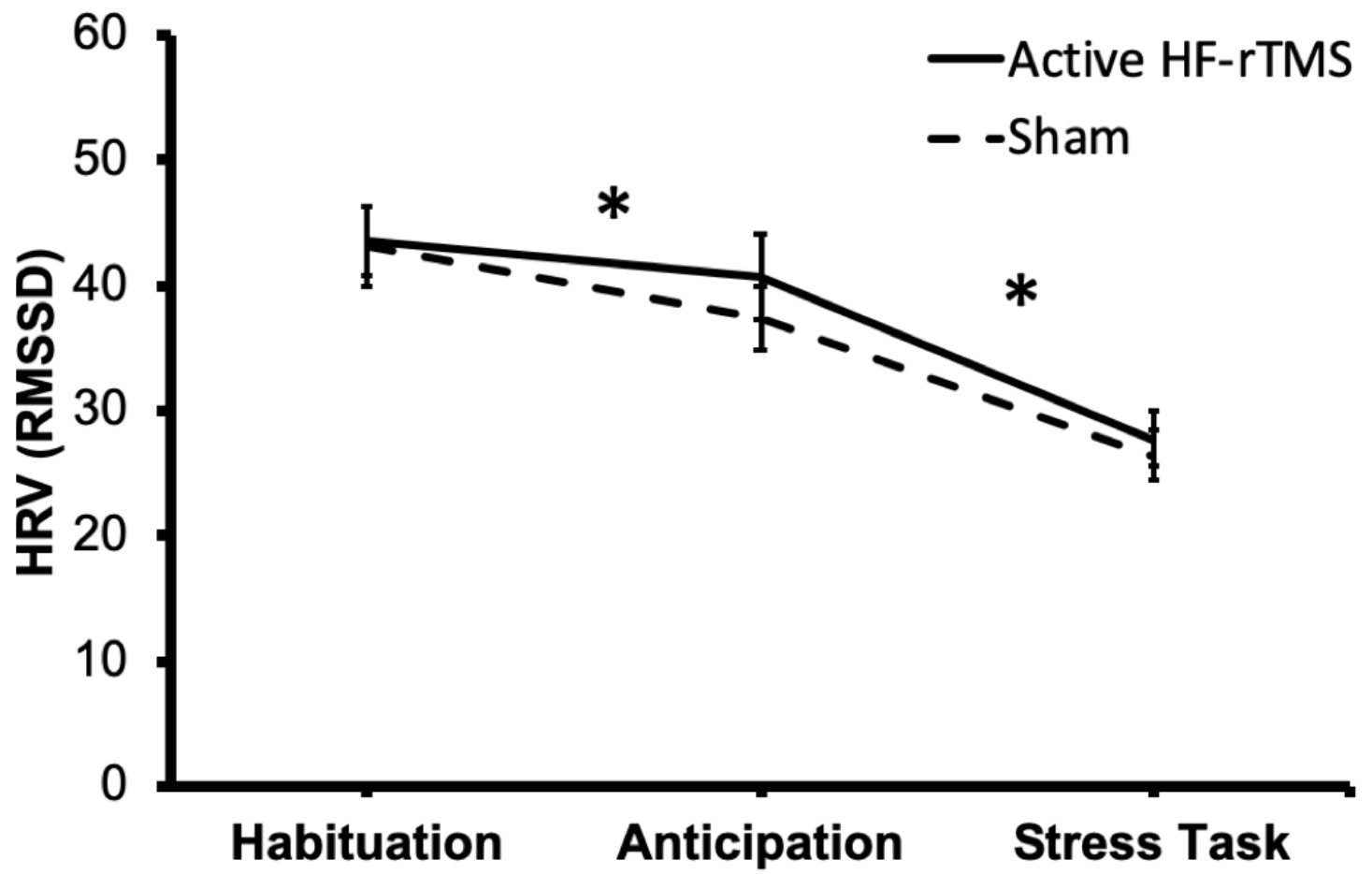


Figure 3
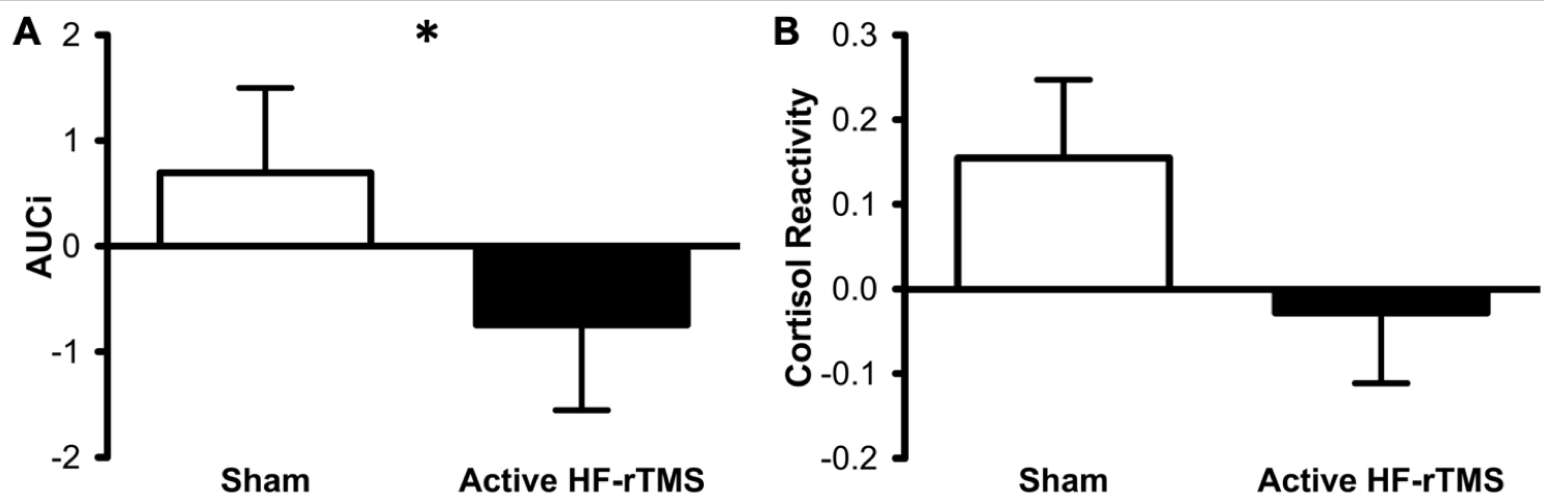